\title{
Applying Fuzzy Logic Principles to Improve the Performance of the Random Early Detection Algorithm
}

\author{
A. I. A. Jabbar, PhD \\ Professor \\ Department of Electrical Engineering \\ Mosul University
}

\author{
Ahmed I. Al-Ghannam \\ M.Sc. Student \\ Department of Electrical Engineering \\ Mosul University
}

\begin{abstract}
This paper proposes a Random early detection algorithm based on fuzzy logic Principles. The main target of using the fuzzy logic is to reduce the number of lost packets which are sent by a sender using RED algorithm in queue-buffer router of the network topology. The function of fuzzy logic is to dynamically tune the maximum drop probability $\left(\max _{\mathrm{p}}\right)$ parameter of the RED algorithm. To realize this target, a twoinput-single-output fuzzy logic is implemented. The inputs of the fuzzy logic are average queue size, the difference in average queue size. To estimate the performance of the FLRED: simple network topology with FTP is suggested. In this research, the opnet modeler 14.5 has been used. The simulation results show that the FLRED algorithm is better than traditional RED algorithm as far as the number of lost packets is concerned.
\end{abstract}

\section{Keywords}

Active queue management (AQM), Congestion Control , Fuzzy logic Random early detection (FLRED), Random early detection algorithm (RED).

\section{INTRODUCTION}

Internet provides continuously many new applications such as Web, multimedia, etc. However, due to lack of estimating interference and the number of users who may access Internet in a given time congestion may occur. The resulted long delays in data transmission may increase the queue length in the buffer of intermediate routers leading to overflow [1]. To prevent router buffer from overflow and to minimize end congestion control active Queue Management (AQM) scheme has been proposed. The major advantage of using AQM scheme is to provide congestion information early enough to the end system, to reduce the transmission rate before the buffer overflows [2]. Many of AQM algorithm has been proposed, the well-known AQM algorithm, random early detection (RED) algorithm. Moreover the RED has been recommended by the Internet Engineering Task Force (IETF) [3]. The basic idea of RED is to decide whether the network is congestion through testing the average router queue length. And when the average length of the queue reaches a certain threshold, a random selection of some of the new arrival packets are forced to be discarded or marked, and inform the source to reduce the congestion windows. The source will reduce the sending rate of packet and relieve congestion RED algorithm can improve the link bandwidth utilization and reduce the average queue length[4]. To improve the performance of the RED algorithm many versions have been proposed such as ARED, SRED, BLUE, etc. this paper proposes an improved algorithm FLRED, which uses fuzzy logic to estimate the maxp of origin RED algorithm depending on the average queue size and the difference between any two average queue sizes.

\section{THEORY}

\subsection{Conventional Random Early Detection algorithm}

This algorithm can solve the problem of lock out and global synchronization in passive queue management, and prevents the congestion problem before the buffer over flow. The disadvantage of this algorithm is related to the sensitivity of the parameters which must be chosen carefully. RED discards the packets which arrives the router by the average queue length. It is composed of two independent algorithms. The first one deal with calculation of the average queue length while the second algorithm is designed to calculate packet dropping/marking probability which determine the router at the current congestion level to drop/mark packets according to the value of the calculated probability [4].

\subsection{Mathematical analysis of conventional RED}

The average queue size of a router is a function of the period when the queue is empty (the idle period), the number $(\mathrm{m})$ of small packets that may be transmitted by the router during the idle period. After that the router calculates the average queue size as if the $m$ packets had arrived to the queue during the idle period. The calculation of the average queue size is based on an exponentially weighted moving average (EWMA), as shown in equation 1 :

avg $=$

$$
\left\{\begin{array}{rr}
(1-W q) \times a v g+W q \times q & \text { if } q>0 \\
(1-W q)^{m} \times a v g & \text { otherwise }
\end{array}\right\}
$$

Where:

avg average queue size.

q the current queue size.

Wq a queue weight, $0 \leq \mathrm{Wq} \leq 1$.

$\mathrm{m}$ a number estimated by some function (during the idle period).

Then the average size of the queue is compared with minimum threshold $\left(\min _{\mathrm{th}}\right)$ and maximum threshold $\left(\max _{\mathrm{th}}\right)$, see figure(1). If the value of average queue size is less than $\min _{\text {th }}$, then the packet is placed on the queue. If the value of 
average queue size is equal to or greater than $\max _{\mathrm{th}}$, then the congestion is assumed to be existed and the arrived packet is discarded immediately. If the average size of the queue is in between $\min _{\mathrm{th}}$ and $\max _{\mathrm{th}}$, then congestion is expected to be occurred. At this case, the temporary probability $(\mathrm{Pb})$ can be calculated. as shown in equation 2:

$$
p b=\max _{p} \times \frac{a v g-\min _{t h}}{\max _{t h}-\min _{t h}}
$$

Where :

$$
\max _{\mathrm{p}}=\text { the maximum drop probability. }
$$

Finally, the actual drop probability (pa) is calculated using equation 3:

$$
p a=\frac{p b}{(1-\text { count } \times p b)}
$$

Where:

count $=$ the number of packets which are arrived consecutively and not discarded since the last marked packet.

The Pa increases slowly as the count increases starting from the last marked packet. This ensures that the router does not wait too long before marking a packet.

It should be noticed that RED algorithm depends on Queue Weight $\left(\mathrm{w}_{\mathrm{q}}\right)$, Maximum drop probability $\left(\max _{\mathrm{p}}\right)$, Maximum threshold (max thresh) and Minimum threshold (min thresh).

The optimal performance depends on the way of choosing the four parameters of the algorithm successfully [5].

\subsection{Fuzzy logic}

The fuzzy logic interference is the main unit of a fuzzy logic system, see figure (2) [6]. This paper chooses Mamdani's fuzzy inference method which is based on the following requirements:
1. a set of fuzzy rules

2. Inputs membership functions (includes the process of changing crisp values into forms suitable for linguistic terms of fuzzy sets).

3. Fuzzy rules to create a rule strength from the fuzzifying inputs.

4. combining the rule strength and the output membership function to find the consequences of the rules.

5. Combining the consequences to get the output distribution

6. Defuzzifying the output distribution class type is needed.

\subsection{Fuzzy Logic Random Early Detection Algorithm (FLRED)}

Fuzzy logic system is designed to dynamically adjust the Maxp parameter of the RED algorithm to reduce the packets number loss that are transmitted. To achieve this target, a twoinput-single-output fuzzy logic system is used. The inputs of the system are 1) average queue size, 2) the difference between the average queue size while the output is the estimation Maxp . For the best performance of the network in terms of delay time and throughput, the difference between the average queue size is taken between every five consecutive values from the average queue size. The membership functions for the first input are four with linguistic variables (Low, Medium, Medium high, high) while for the second input is three with linguistic variables (negative, zero, positive). The output membership functions is six with linguistic variables (zero, small probability, medium probability, medium big probability, medium medium big probability, big probability), See figure (3).

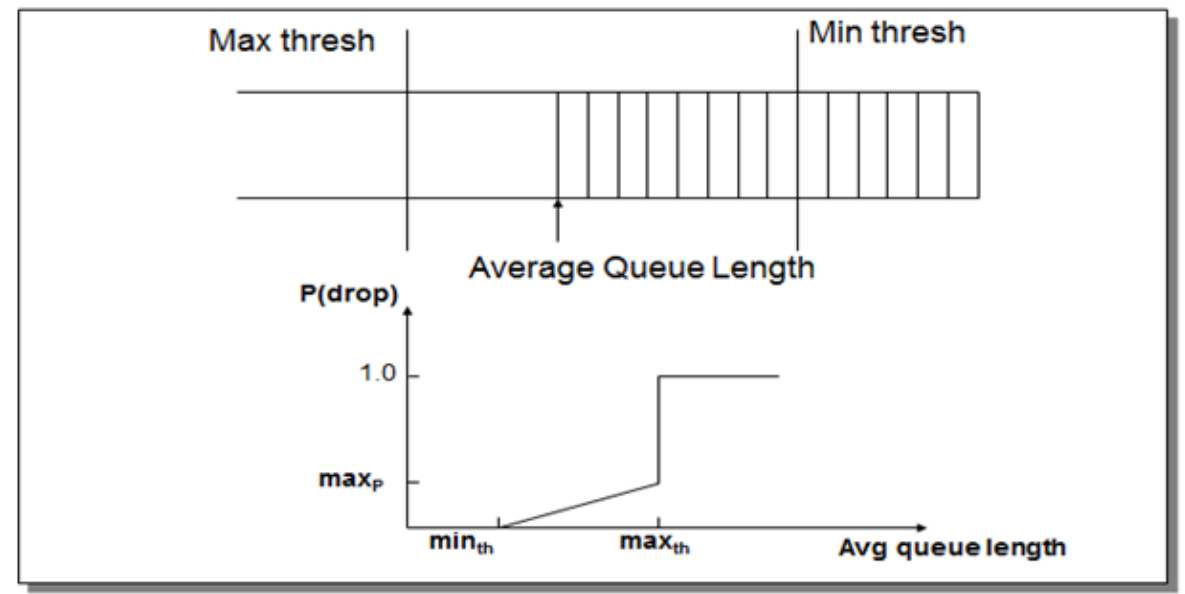

Fig 1: RED algorithm drop function 


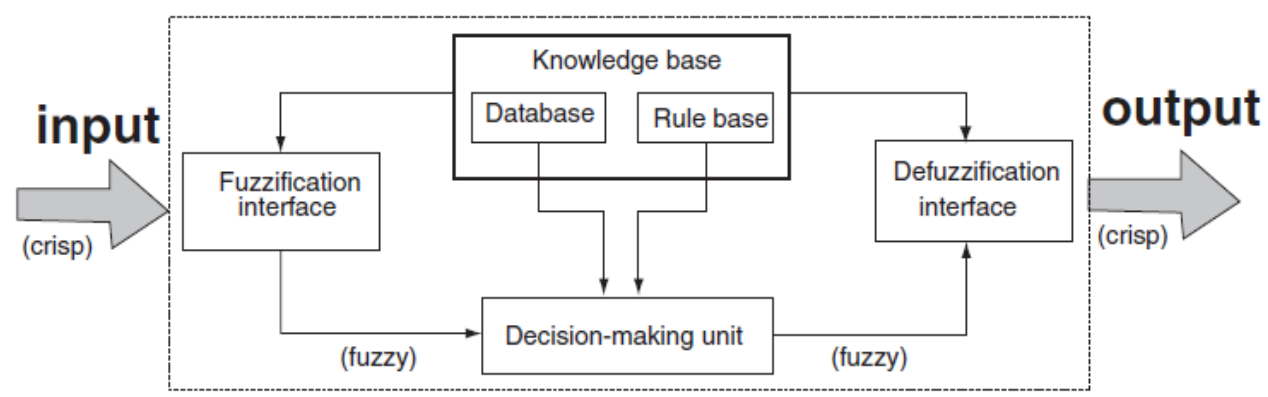

Fig 2: Fuzzy inference system

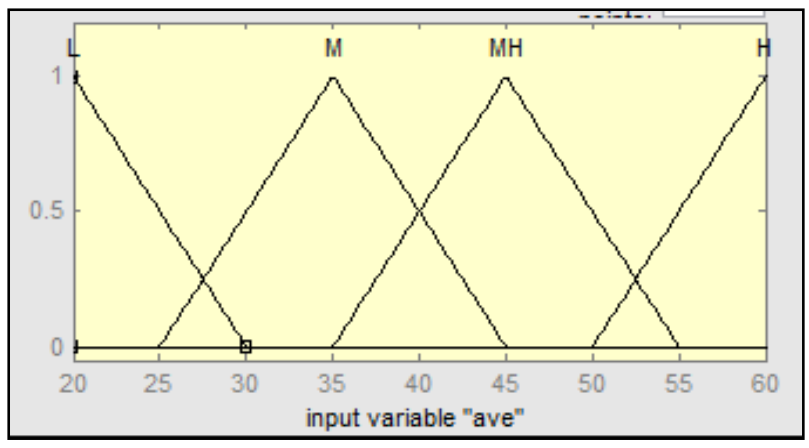

(a) Average queue size

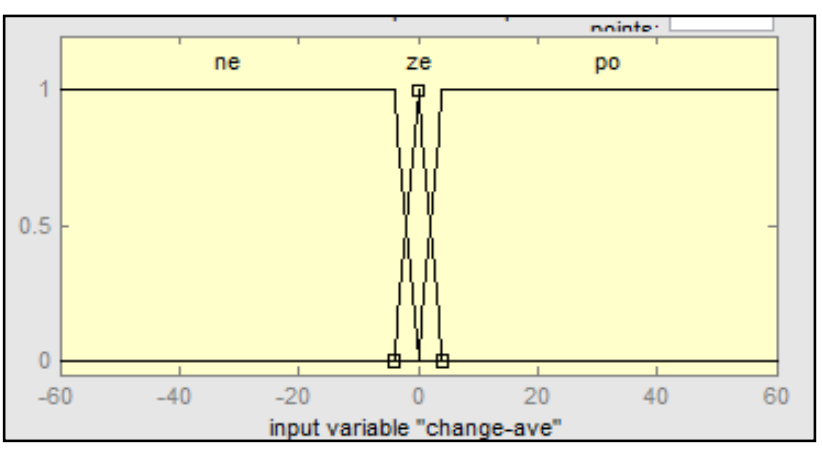

(b) Difference between average queue size

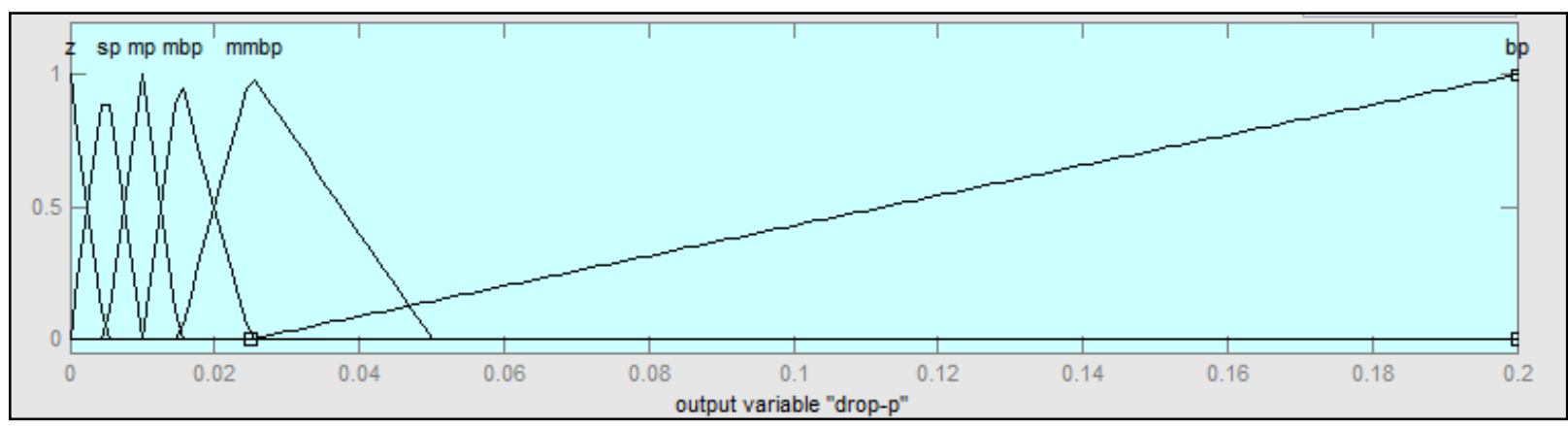

(c) Maximum drop probability

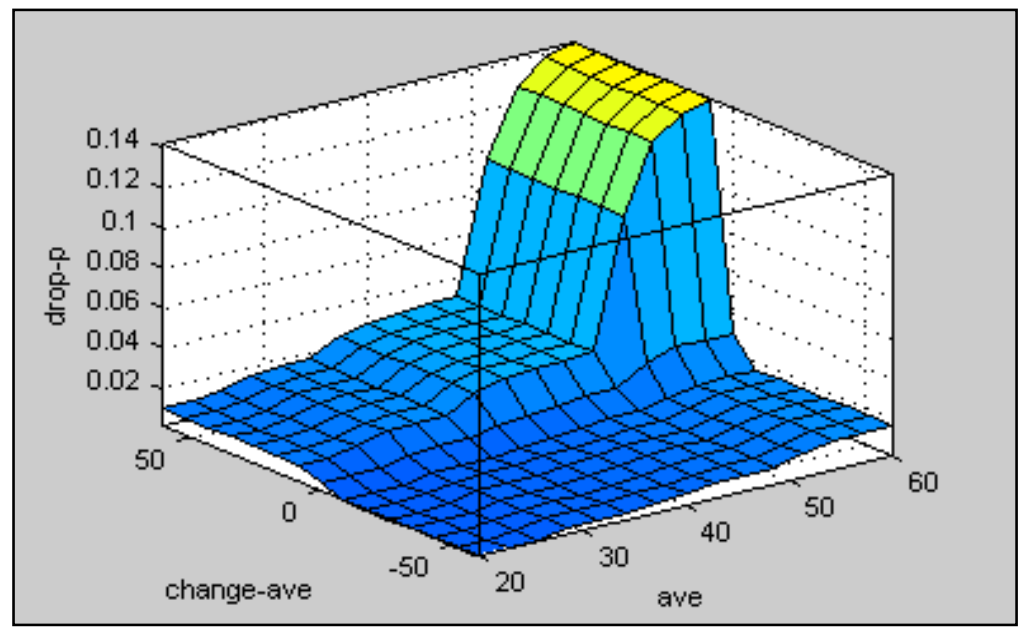

(d) Relationship between input and output

Fig 3: The input and output of the fuzzy logic system 
The rule that is used to map the fuzzy input to the fuzzy output is shown in the Table (1).

Tab 1:The fuzzy logic system rules

\begin{tabular}{|c|c|c|c|}
\hline Rule no. & ave & differ -ave & max $_{p}$ \\
\hline 1 & L & NE & ZE \\
\hline 2 & L & ZE & SP \\
\hline 3 & L & PO & MP \\
\hline 4 & M & NE & SP \\
\hline 5 & M & ZE & MP \\
\hline 6 & M & PO & MBP \\
\hline 7 & MH & NE & MP \\
\hline 8 & MH & ZE & MBP \\
\hline 9 & MH & PO & MMBP \\
\hline 10 & H & NE & MBP \\
\hline 11 & H & ZE & MMBP \\
\hline 12 & H & PO & BP \\
\hline
\end{tabular}

\section{SIMULATION RESULTS}

The obtained results rely on FTP application. using opnet modeler 14.5 [7], the suggested topology is given in figure (4), the RED parameters values that are used in the simulation are $\operatorname{minth}=20$, maxth $=60$, wq=0.002 and maximum buffer size $=70$, While the simulation time $=600 \mathrm{sec}[8]$.

Figure (5) shows the average queue size for both RED and FLRED algorithms of router (1) of the network, While figure(6) shows the packets drop for both RED and FLRED algorithms.

The results declare that the rate of packets loss of the FLRED algorithm is less than the RED algorithm. Where the number of packets dropped in RED algorithm is 1515 packets while in FLRED algorithm is 700 packets. This means that FLRED algorithm dropped packets by $54 \%$ less than RED algorithm.

This is an encouraging result because the FTP application over TCP protocol requires minimum packets loss with relatively acceptable delay, this objective can be achieved by the FLRED algorithm, see figure (7). The figure shows that the difference in queuing delay between the two algorithm (RED and FLRED) is acceptable. The queuing delay increased by $30 \%$ in the FLRED algorithm as compared with RED algorithm.

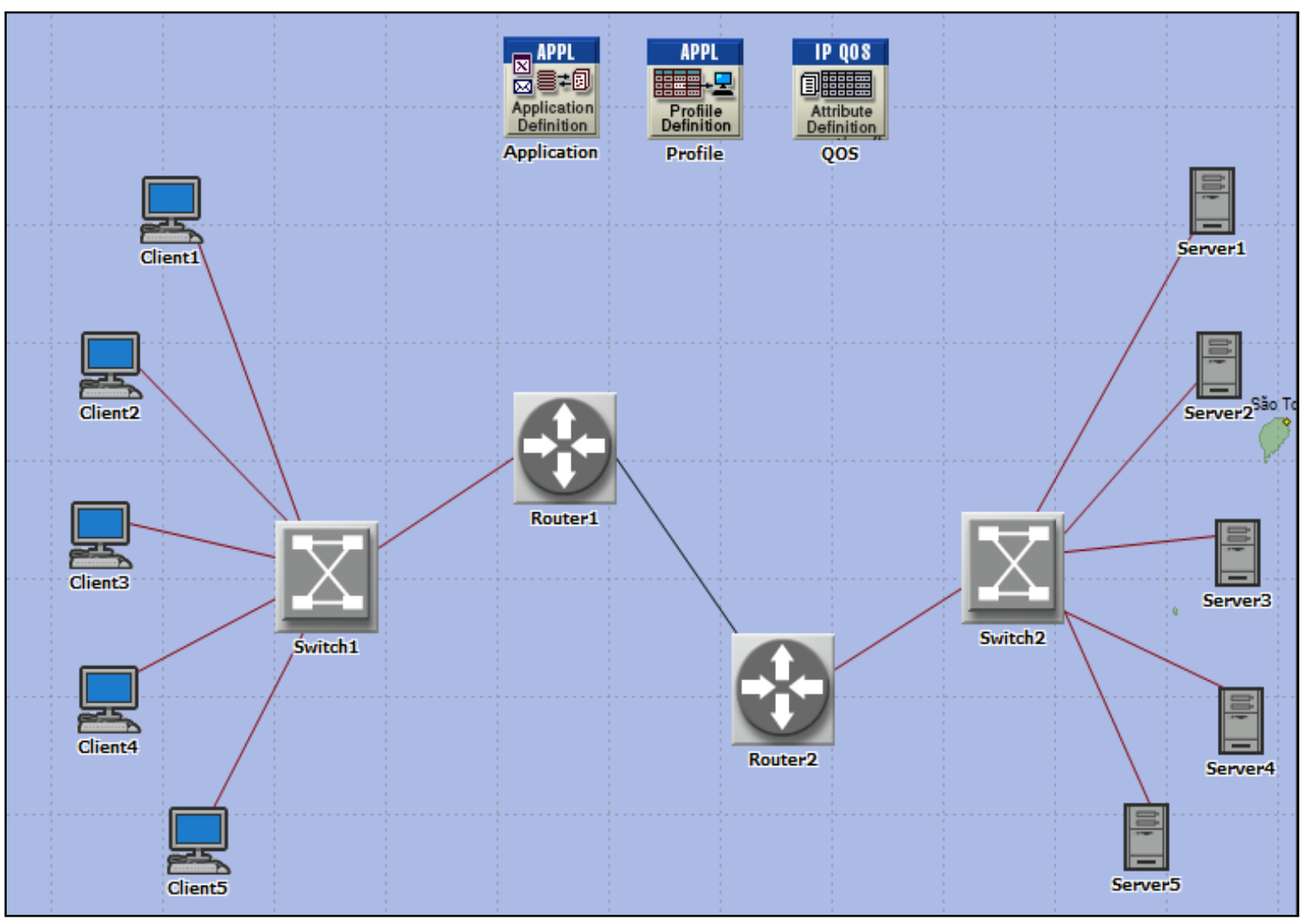

Fig 4: network topology 


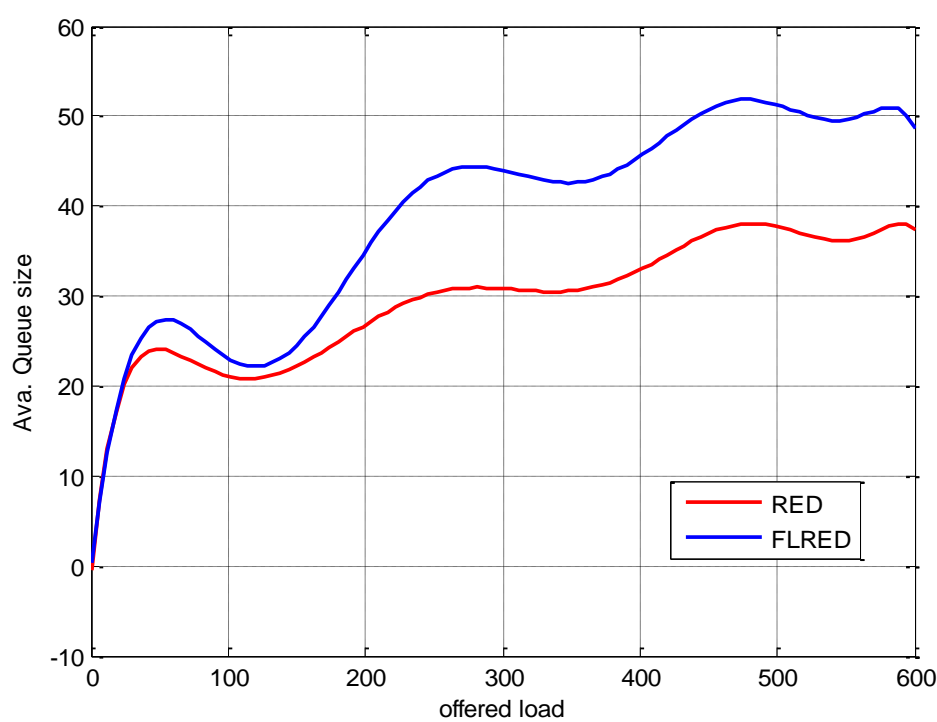

Fig 5:average queue size for both RED and FLRED algorithm

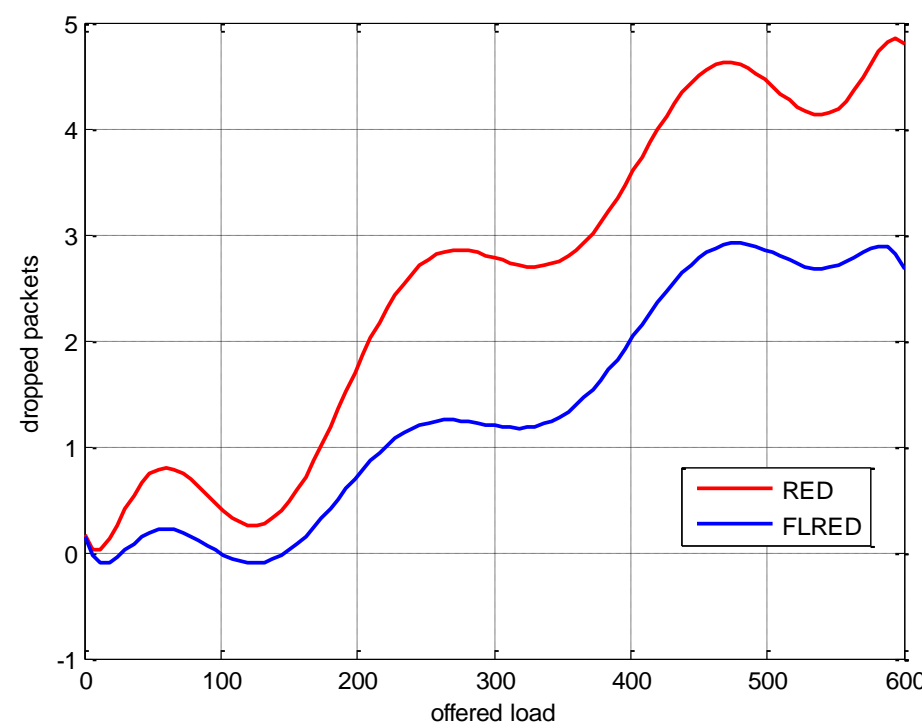

Fig 6: packets drop per second for both RED and FLRED algorithm

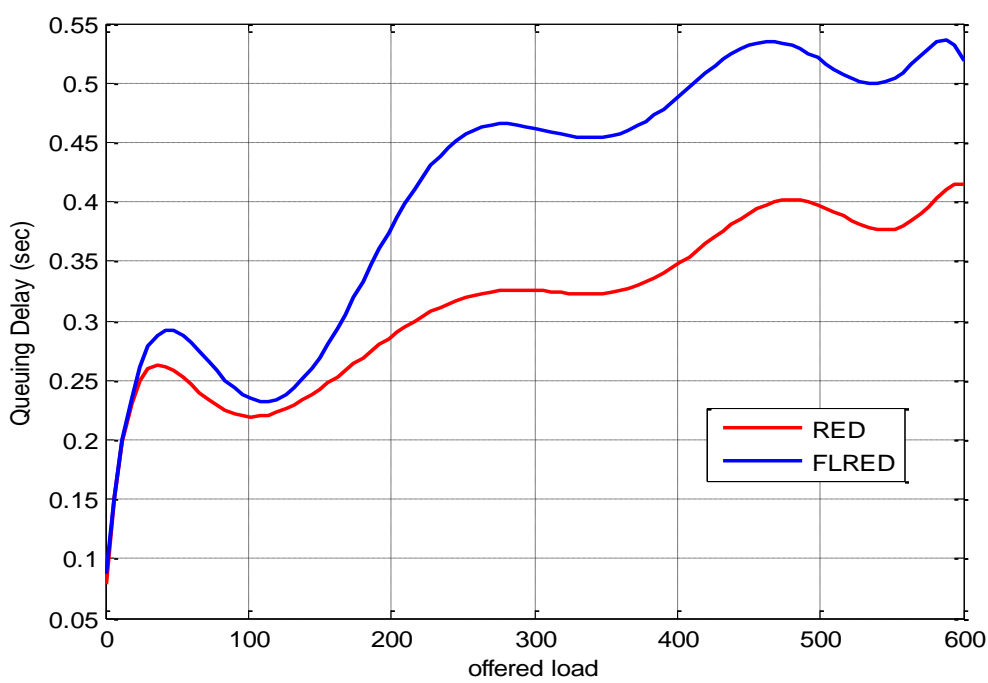

Fig 7: queuing delay for both algorithms (RED and FLRED)

\section{CONCLUSION}

This research proposed a fuzzy logic system which is suitable for different network load and traffic conditions. The proposed algorithm reduces the number of lost packets when FTP application is used. Consequently, the number of retransmitted packets will be minimized. Leading to an improvement of the probability of congestion in the network. finally in next focus of the study, it is use the proposed algorithm in another application.

\section{REFERENCE}

[1] H. ASHTIANI, H. Moradi and M. NIKPOUR . " Active Queue Management in TCP Networks Based on FuzzyPid Controller" , Journal of Applied Computer Science \& Mathematics, 2012.

[2] Minjuan Cheng and Xiaoming Ma, " Performance Evaluation of Queue Management Methods for Congestion Control" , Journal of Information \& Computational Science, 2012.
[3] Arash Dana and Ahmad Malekloo, "Performance comparison between Active and Passive Queue Management" IJCSI International Journal of Computer Science Issues, 2010 .

[4] Jingjun Zhanga, Wenlong Xub and Liguo Wangb, " An Improved Adaptive Active Queue Management Algorithm Based on Nonlinear Smoothing", Sciverse science direct, 2011.

[5] S.Floyd and V.Jacson, Random Early Detection gateways for congestion avoidance, IEEE/ACM Trans.on Networking, 1993.

[6] S. N. Sivanandam, S. Sumathi and S. N. Deepa , "Introduction to Fuzzy Logic using MATLAB" , Springer-Verlag Berlin Heidelberg 2007.

[7] OPNET Technologies, Inc. http://www.opnet.com

[8] T. Álvarez, V. Álvarez and L. Nicolás, "Understanding congestion control algorithm in TCP using OPNET", 2nd International Conference on Education and New Learning Technologies, 2010. 